\title{
Concepção e desenvolvimento de sistema interativo para o aperfeiçoamento da gestão de Comunidades que Sustentam a Agricultura no Distrito Federal Conception and development of an interactive system for the management improvement of Community Supported Agriculture in Distrito Federal
}

\author{
Sofia Santos, Camila Lombardi Torres \& Rogerio Camara
}

Comunidade que Sustenta a Agricultura, sistema interativo, gestão do conhecimento, método

\begin{abstract}
Concepção de sistema interativo que possibilita o registro de experiências e troca de conhecimentos entre Comunidades que Sustentam a Agricultura (CSAs) do Distrito Federal. Apresenta o projeto como meio para se atender a necessidades diagnosticadas no que se refere a maior integração entre participantes das atividades de gestão das comunidades, seus processos cotidianos e ao uso de ferramentas que facilitem o registro e divulgação de informações emergentes. O método aplicado se fundamenta nas obras de Milton Santos, Christopher Alexander, Nathan Sheddroff e de Jesse Garrett, e contempla: levantamento de dados em documentos e em campo; interações com membros de CSAs do DF, com a Rede CSA Brasília e com pesquisadores afins; definição e atribuição de significados a padrões de ações cotidianas das CSAs; desenvolvimento de site com base na análise de conteúdos e funcionalidades atribuídos ao sistema; estudos de interface e validação com participação de usuários; concepção da interface desktop e definição do projeto gráfico do sistema. Por fim, são discutidos os meios de se alcançar a estabilidade do artefato criado, critérios para sua evolução e a apropriação do sistema pela Rede CSA Brasília e usuários finais. Estima-se que o sistema auxilie na implementação de novas CSAs e que seja reaplicado em outras CSAs para além do DF.
\end{abstract}

\section{Community Supported Agriculture, interactive system, knowledge management, process}

Describes the design process of a interactive system whose objective is to enable the registration of experiences and knowledge exchange between the Communities that Support Agriculture (CSA) in Distrito Federal. The project aims to meet the diagnosed needs of greater integration among participants in community management activities, their day-to-day processes and the use of tools that could improve the registration and dissemination of emerging information. The method applied is based on the works of Milton Santos, Christopher Alexander, Nathan Sheddroff and Jesse Garrett, and includes: data collection in documents and in the field; interactions with CSA members, Rede CSA Brasilia and related researchers; definition and attribution of meanings to CSA daily action patterns; the development of a website based on the analysis of contents and functionalities attributed to the system; interface studies with participation of users; and definition of the system's graphic design. Lastly, the means of achieving the stability of the artifact, the criteria for its evolution and the appropriation of the system by both the Rede CSA Brasilia and its end users are discussed. It is estimated that the artifact developed can aid in the implementation of new CSAs and that it can be reapplied in other cases beyond DF's.

\section{Introdução}

A Comunidade que Sustenta a Agricultura (CSA) é um modelo de produção e distribuição de produtos agrícolas que preza pelo contato direto entre consumidores e produtores. Os consumidores - chamados de coagricultores - concorrem com os riscos e responsabilidades junto aos agricultores e, em troca do financiamento, periodicamente recebem produtos agrícolas colhidos pelos produtores. Dada a ausência de intermediários nas CSAs, há a necessidade de que os consumidores e produtores promovam, eles mesmos, a administração e a distribuição da produção coletiva.

Para a organização e administração de uma CSA, é necessário que seus membros adquiram conhecimentos sobre a prática de princípios que fundamentam o funcionamento do modelo, compreendendo as experiências daqueles que viveram ou vivem a dinâmica deste tipo

Anais do 9 CIDI e 9 CONGIC

Luciane Maria Fadel, Carla Spinillo, Anderson Horta, Cristina Portugal (orgs.)

Sociedade Brasileira de Design da Informação - SBDI

Belo Horizonte | Brasil | 2019

ISBN 978-85-212-1728-2
Proceedings of the 9th CIDI and 9th CONGIC

Luciane Maria Fadel, Carla Spinillo, Anderson Horta, Cristina Portugal (orgs.)

Sociedade Brasileira de Design da Informação - SBDI

Belo Horizonte | Brazil | 2019

ISBN 978-85-212-1728-2 
de comunidade. Tais referências existem, em maioria, apenas enquanto conhecimento tácito, carecendo de registros estruturados, limitando sua propagação. Este projeto objetivou possibilitar o registro de informações que residem no plano tácito, facilitar a troca de conhecimentos e ampliar o acesso às práticas existentes em CSAs do Distrito Federal, com a finalidade de instruir e possibilitar o fortalecimento da gestão comunitária. Para tanto, visou-se a criação de sistema interativo com interface responsiva, capaz de ser operada no cotidiano dos agentes envolvidos, promovendo o registro e a troca de informações sobre o funcionamento das comunidades.

Além do entendimento sobre o conceito e a prática das CSAs por meio de debate junto a pesquisadores e membros da equipe projetista que participam de CSAs, de visitas ao site da Rede CSA Brasília e da leitura de textos e dissertação sobre o tema (Torres, 2017), outros conceitos que fundamentaram pesquisa e criação foram: arquitetura da informação (Shedroff, 1999); conhecimento tácito (Lassance Jr. e Pedreira, 2004); linguagens de padrões (Christopher Alexander, 2013); estrutura, função, processo e forma (Santos, 1992); gestão do conhecimento (Takeuchi e Nonaka, 2008); rede de atores (Callon, 1987); e Tecnologia Social (Bava, 2004; Dagnino et al, 2004).

Procurou-se prospectar o modo de inclusão de um sistema interativo na dinâmica das CSAs em um projeto que contou com a participação de vinte pessoas, entre pesquisadores voluntários, bolsistas de iniciação científica e de apoio técnico, de diferentes áreas do conhecimento (design, antropologia, psicologia, saúde, ciências da computação, engenharia de software) e explorou-se os diversos temas em grupos formados a partir do interesse e de competências dos membros da equipe.

Recorreu-se, para a ideação e definição do sistema, ao método de desenvolvimento de mídia interativa com foco na experiência do usuário proposto por Jesse James Garrett (2011). A referência foi fundamental para a documentação e evolução do projeto, especialmente no que diz respeito à análise concomitante dos objetivos do sistema e das necessidades dos usuários, à especulação de funcionalidades e conteúdos a fim de se solucionar os problemas encontrados, à concepção da narrativa que compõem o design de interação e dos elementos necessários à interface, à compreensão do usuário sobre as informações contidas no sistema, e à participação de usuários ao longo do processo.

\section{Objetivos do sistema e necessidades dos usuários}

A proposta inicial de desenvolvimento do sistema interativo surgiu dos resultados da dissertação de mestrado em design de Camila Torres (2017) e de temas relativos à qualidade de formação e funcionamento de CSAs, com destaque a dificuldades encontradas nas dinâmicas autogestionárias, que se dão conforme a disponibilidade de seus membros e à necessidade de se aproximar conhecimentos emergentes das comunidade. Com o objetivo de viabilizar a produção tecnocientífica, a proposta foi submetida e aprovada em edital da Fundação de Apoio a Pesquisa do Distrito Federal (FAP-DF). Dentre os objetivos específicos do projeto, destaca-se: investigar formas de articulação e produção, e deslocamentos geográficos no cotidiano das CSAs; identificar processos de comunicação, dinâmicas sociais (atores, processos, fluxos, problemas recorrentes e soluções criativas) e os padrões de gestão; desenvolver e tornar acessível um sistema interativo que abrigue possibilidades de difusão e gestão da informação, compartilhamento de notificações, assim como a criação de interfaces adequadas frente à diversidade instrucional dos membros das CSAs.

Com base no diagnóstico preliminar e nos objetivos da primeira etapa de projeto proposta por Garrett (2011) (i.e. Definição de Estratégia), realizou-se novo levantamento sobre necessidades dos usuários, considerando fatores anteriormente não identificados. Recorreu-se a dados da pesquisa "Perspectivas da evolução das CSAs em Brasília"1, de Aleksia Boon, a depoimentos dos agricultores das CSAs do DF, e a atas de encontros realizados pela Rede

\footnotetext{
1 Título provisório e traduzido livremente pela autora de um estudo à época em fase de desenvolvimento.
} 
Santos, S., Torres, C. L. \& Camara, R. | Concepção e desenvolvimento de Sistema Interativo para o aperfeiçoamento da gestão de Comunidades que Sustentam a Agricultura no Distrito Federal

CSA Brasília ${ }^{2}$. Os resultados foram posteriormente sistematizados (concepção e relação de objetivos conforme necessidade diagnosticada).

A definição de estratégia considerou necessidades a partir de ações elementares ao funcionamento de uma comunidade. Muitas vezes o que se declara como 'necessidade' parte da observação de sintomas de problemas que a precede, sendo este último passível de análises que podem colaborar para soluções de maior impacto. Buscou-se compreender o espaço sociotécnico a partir de sua estrutura, funções e processos (Santos, 1992), como meio de se identificar oportunidades de melhorias da dinâmica autogestionário implícita a uma CSA. Compreendeu-se na prática o conceito de CSA e seus princípios a partir da observação em campo e coletou-se dados em conversas, registro fotográfico, uso de caderneta de campo e diários etnográficos.

Como síntese da coleta em campo, realizou-se diagrama de relações entre fatores que representam a operação das CSAs, considerando regularidades cotidianas e das ações sistêmicas. O procedimento resultou em exercício de arquitetura da informação do sistema que explicita a dinâmica das CSAs, considerando estruturação, ordenação e classificação de dados. O tipo de dado abordado foi compreendido como um padrão, conforme conceito explorado pelo Christopher Alexander ${ }^{3}$ (2013). No registro dos padrões de ação das CSAs, foram reconhecidas ações que acontecem em qualquer comunidade, a fim de que as soluções encontradas fossem reconhecíveis.

$\mathrm{Na}$ busca do que há em comum, observou-se os eventos recorrentes. O pagamento de cotas, por exemplo, acontece em todas as CSAs do DF, porém seu modo de realização pode se diferenciar a depender de decisões comunitárias. Foram levantadas aproximadamente quarenta ações-padrão das CSAs do DF e especificou-se os modos de realização.

As ações-padrão foram organizadas considerando as maneiras que se relacionam numa rotina - o pagamento de cestas, por exemplo, pode acontecer antes, depois ou simultaneamente à outra ação. O processo revelou uma suposta estrutura implícita do sistema, que ressaltou tanto as ações inerentes ao funcionamento de uma CSA do DF, bem como a compreensão de interfaces e de interações entre os eventos recorrentes (Imagem 1).

\footnotetext{
2 Entidade autônoma e sem fins lucrativos, movida por voluntários, cuja missão é "ser o elo de integração e fortalecimento do movimento social de Comunidades que Sustentam a Agricultura no DF, para promover uma cultura solidária, saudável e sustentável de produção e consumo de alimentos" (REDE CSA BRASÍLIA, 2017)

3 Para Alexander, um padrão representa um elemento capaz de se desdobrar em diversas facetas, porém sem perder sua natureza essencial. O padrão funciona como parâmetro, a partir do qual se observa possíveis variáveis. Ele ainda adiciona que o que caracteriza um determinado local são os padrões de evento que acontecem repetidamente neste.
} 
Imagem 1: Diagrama da estrutura implícita revelada das CSAs do DF

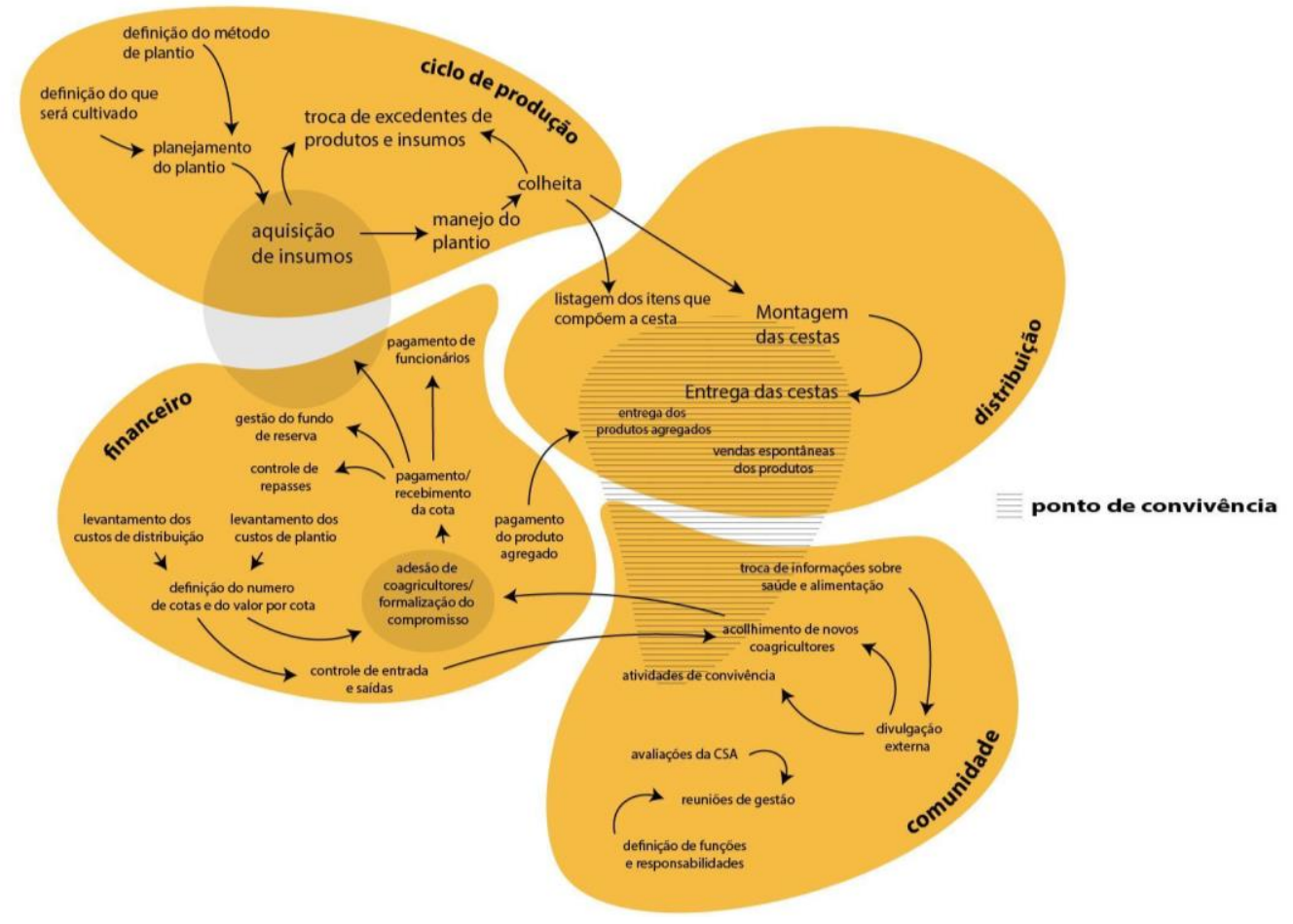

Cada padrão foi definido conforme características listadas abaixo:

- Atores envolvidos: atores de determinado padrão;

- Descrição: maneira como o padrão se dá, sem especificar qualquer possibilidade de variação do mesmo:

- Variações/Modos de realização: as formas possíveis de um mesmo padrão desdobrarse, sem que mude sua natureza essencial;

- Lugar: expressão espacial relacionada ao padrão, não necessariamente uma localização geográfica;

- Periodicidade: período de tempo decorrente do exercício de determinado padrão;

- Informação gerada: ação que pode ser registrada ou comunicada a um terceiro;

- Meio de transmissão da informação gerada: mídias utilizadas;

- Relações com outros padrões: lista de ações mapeadas que tem interface com o padrão ora definido.

A partir da construção da arquitetura da informação compreendeu-se a complexidade da gestão das CSAs; detalhou-se a tabela de objetivos do sistema e necessidades dos usuários; as interfaces entre os padrões suscitou especulações sobre funcionalidades e motivações do usuários; e sua documentação tornou-se rascunho para conteúdos textuais do sistema. Após análise dos resultados, fez-se uma síntese das necessidades e uma lista representativa do público-alvo, respectivamente descritos abaixo:

- Principais necessidades:

a. Aperfeiçoar processos de autogestão das CSAs;

b. Informar coagricultores e interessados em CSAs;

c. Integrar mais coagricultores na autogestão;

d. Possibilitar a troca de informações sobre práticas de gestão e de produção;

e. Melhorar a divulgação das CSAs para atrair novos coagricultores. 
Santos, S., Torres, C. L. \& Camara, R. | Concepção e desenvolvimento de Sistema Interativo para o aperfeiçoamento da gestão de Comunidades que Sustentam a Agricultura no Distrito Federal

- Público-alvo:

a. Agricultores e coagricultores experientes (i.e. aqueles que participam ativamente de atividades de gestão da comunidade e que buscam referências para otimizar processos);

b. Novos agricultores e coagricultores (i.e. recém membros que precisam de informações para compreender o funcionamento de sua CSA);

c. Gestores da Rede CSA Brasília (i.e. atores que zelam pelas boas práticas dessa tecnologia social e buscam meios de formação de novas comunidades);

d. Pessoas curiosas (i.e. interessados em se tornar agricultores ou coagricultores)

e. Pesquisadores (i.e. considera-se os constantes estudos científicos sobre o tema)

O processo foi apresentado a representantes da Rede CSA Brasília, que retornou com suas percepções e ideias sobre conteúdos e funcionalidades do sistema. Participantes dessa apresentação, além de outros atores de CSAs do DF, foram posteriormente convidados a participarem de cardsortings com o intuito de se investigar os modos de organização de conteúdos previstos para o sistema e de promover a ideação espontânea de outros atributos.

\section{Definição das funcionalidades e da estrutura do sistema interativo}

O diagrama revela ações que acontecem nas CSAs do DF, porém a maneira como acontecem varia de acordo com a comunidade observada. Logo, compreendeu-se que a estrutura primária se desdobra em outras estruturas específicas com atributos de funcionalidade. As açõespadrão foram oferecidas aos usuários do sistema experientes em CSA, que podem reconhecer o evento em suas próprias comunidades devido ao caráter genérico de cada ação-padrão. Ao usuário é solicitado informar como aquela ação-padrão acontece em sua comunidade, evidenciando particularidades, a periodicidade e o modus operandi. Busca-se oportunizar o compartilhamento de soluções e de práticas do dia-a-dia da CSAs, de maneira orgânica, caracterizando modos de ação que são classificados pelo sistema e correlacionadas.

O sistema contempla ainda a disponibilização de modelos de documento para aprofundamento e melhor registro de informações decorrentes do exercício da prática em uma CSA, área para discussão sobre as práticas, apresentação eficiente e detalhada das CSAs do DF, ambiente para registros internos e particulares de uma CSA, além de conteúdo estático sobre o que é CSA e links para conteúdos afins.

Definiu-se o artefato como sistema web responsivo, com adequação de conteúdo para plataformas desktop e móveis, facilitando o acesso à visualização e inclusão de informações no cotidiano dos diversos agente envolvidos. O Design de Interação foi estruturado em forma de diagrama, relacionando conteúdos e ações dos usuários. A estrutura priorizou a relação sistêmica triangular entre os dados inscritos como perfil de uma CSA (lista de comunidades), os verbetes representativos das práticas (similar a proposta de wikis) e fóruns demarcados por esses verbetes. Este diagrama foi a principal referência para a concepção da interface (Imagem 2). 
Santos, S., Torres, C. L. \& Camara, R. | Concepção e desenvolvimento de Sistema Interativo para o aperfeiçoamento da gestão de Comunidades que Sustentam a Agricultura no Distrito Federal

Imagem 2: Detalhe do diagrama que estrutura o Design de Interação.

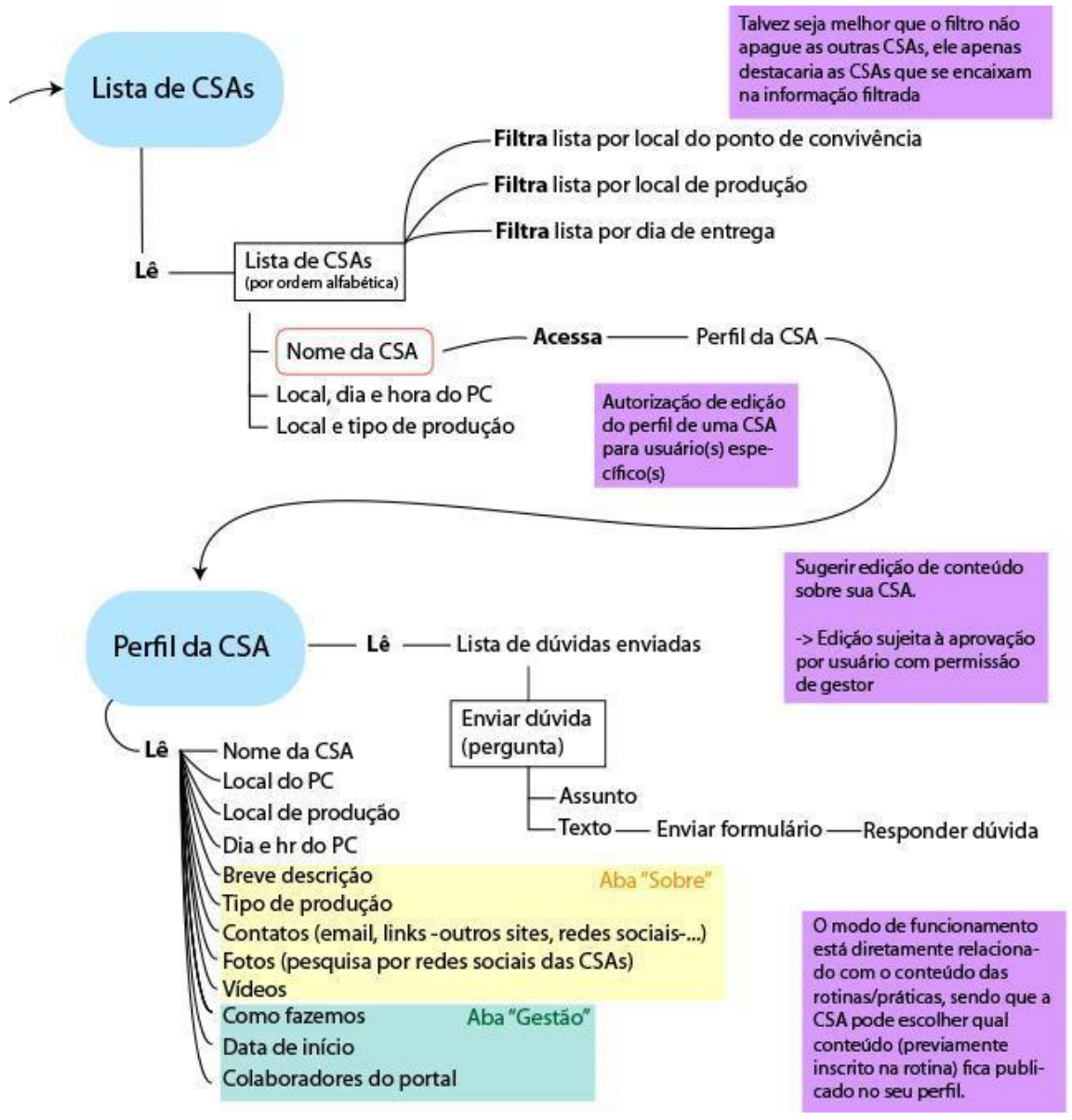

\section{Desenvolvimento do site responsivo}

Definidas as áreas do sistema, a equipe de desenvolvimento avaliava variáveis integrativas. Foi realizado um estudo de soluções para a programação de backend e de frontend com foco na eficiência da relação entre dados e sua visualização. Considerou-se a criação de um CMS (Content Management System) para melhor gerenciamento de conteúdo, na intenção de que membros ativos de CSAs possam, de forma autônoma, realizar as adequações necessárias.

A definição do projeto gráfico se deu paralelamente aos resultados de testes com a versão smartphone, incluindo avaliações colhidas por usuários em momentos de pesquisa de usabilidade em campo com uso do protótipo navegável. Após a definição de interface da maioria das telas da versão móvel, se iniciou a adequação da interface para o acesso em plataforma desktop (Imagens 3 e 4). 
Santos, S., Torres, C. L. \& Camara, R. | Concepção e desenvolvimento de Sistema Interativo para o aperfeiçoamento da gestão de Comunidades que Sustentam a Agricultura no Distrito Federal

Imagem 3: Exemplo 'Perfil da CSA' na versão smartphone.
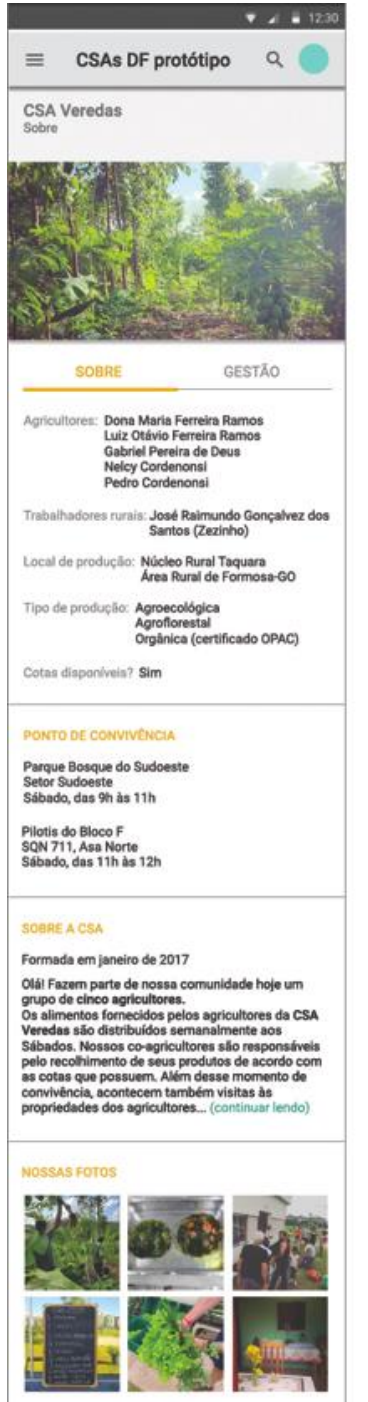

ver mais fotos

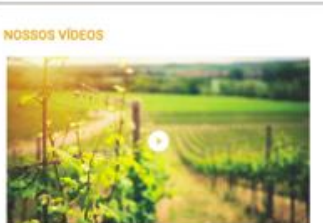

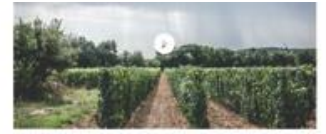

ver mais videos
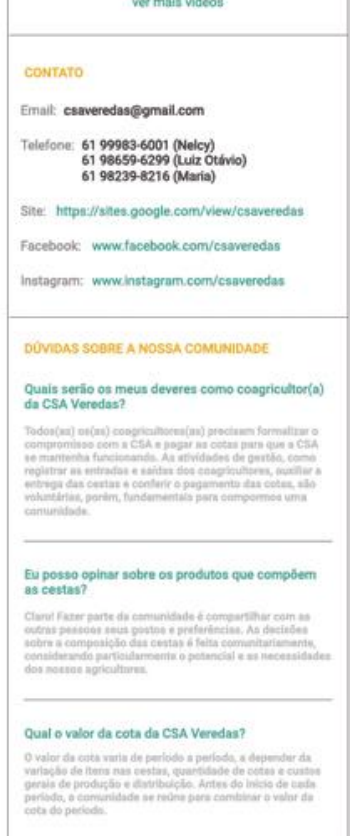

Euposso particicipar da CSA Verodas como
agicultor(a)?

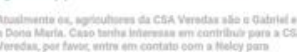

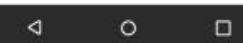

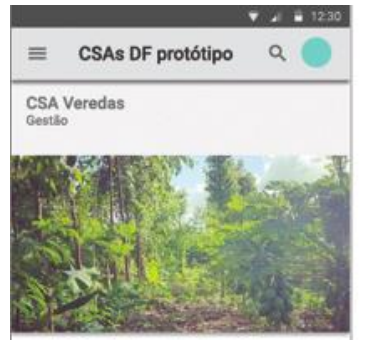

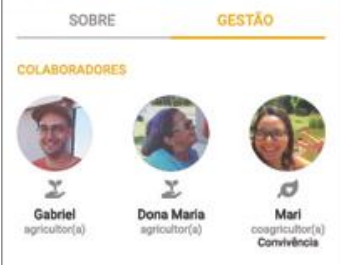

COMO FAZEMOS

Cielo de Producibio:

Manejo do Plantio

Com a participaçio de cospricultores: multirbes
evisiths espontineas ocasionalmente

Podas do extrato mais atho

ocasionaimente
na agroforesta

Comissbo do Financeirox:

Controle do pagamento das cotas

Conferencila de comprovantes bancirios

mensalmente
poremal

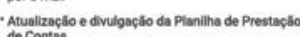
mensalmente
na planiha digtal

1. Planilha de Prestachio de Contas xits

evartamento dos custos de produç co e distribuiche

- Atuastracho do Quadro de linvestimentos da
Producfio

ccasionsthente
na planilha doglat

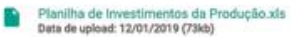

caniento

Comists bo do Acolhimento:

Achento de novos coagricultor Atualizacito da ista de presencan
anualmente e/ou ocssionamente na planiha digtai

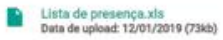

-Encaminhar e rececter os Termos de Compromisso

anualmente e/ou ocasionaimente
no Ponto de Convivencia e/ou por email

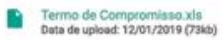

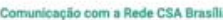

Contatos com outras CSAS

ocasionaimente
por Whatsapp

- Participoçso em reunizes da CSA Braslita

Comissbo de Comuniceacho

Divilgacho imterens

Divulosecho de informaçees sobre temas de

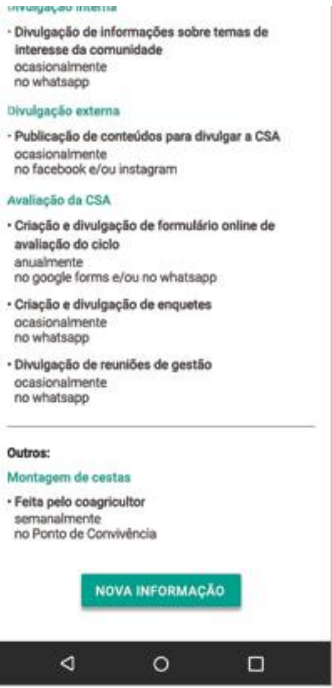

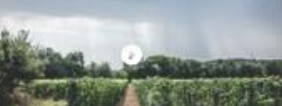


Santos, S., Torres, C. L. \& Camara, R. | Concepção e desenvolvimento de Sistema Interativo para o aperfeiçoamento da gestão de Comunidades que Sustentam a Agricultura no Distrito Federal

Imagem 4: Exemplo 'Perfil da CSA' na versão desktop.

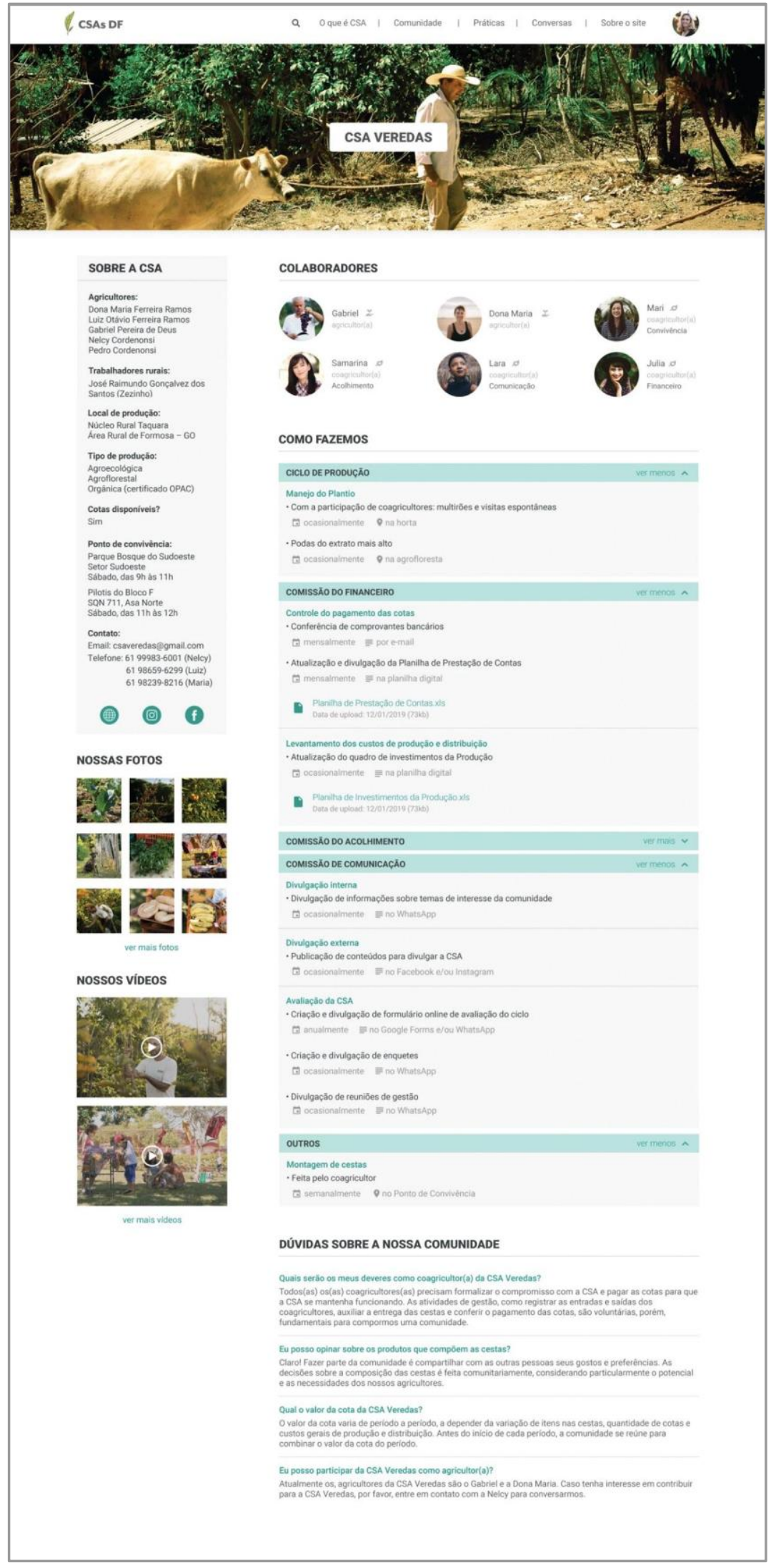

Anais do 9 Congresso Internacional de Design da Informação | CIDI 2019 


\section{Considerações finais}

Devido à complexidade do sistema fez-se necessário a contratação de uma empresa parceira com foco em desenvolvimento de sistemas web. A lista de tarefas atribuídas a estes contemplou manutenção incremental e corretiva do sistema pelo período de seis meses, entendendo-se por incremental a implementação de funcionalidades de alta complexidade (sistema interno de busca/pesquisa e visualização gráfica e lúdica dos dados). Já a manutenção corretiva visa a busca por estabilidade do sistema decorrente de ajustes a problemas que potencialmente vão se apresentar.

O sistema proposto, em fase de implementação final, responde a demandas sociais concretas; valorizar e favorecer a aprendizagem e a construção de conhecimentos coletivos característicos das CSAs; promove a troca de saberes para o encorajamento de comunidades. Possibilita a sistematização dinâmica do conhecimento gerado e sua veiculação. Ao se prever o aumento de verbetes representativos das práticas e ao se reconhecer a necessidade de resoluções referentes aos níveis de autorização para inserção de informações do sistema em comum acordo com a Rede CSA Brasília, é de suma importância dar-se continuidade ao projeto para além da estabilidade do site publicado. Estão previstos estudos contínuos de usabilidade envolvendo participantes de CSAs do DF, além da capacitação voluntária de membros da Rede CSA Brasília, com o objetivo de assegurar o uso e a constante evolução do sistema.

Sabe-se da ocorrência de demandas semelhantes ao observado em CSAs do DF em outros estados brasileiros e espera-se que o artefato concebido possa ser reaplicado para casos alhures como ferramenta de suporte à gestão coletiva, além de colaborar para a divulgação das comunidades aos interessados em fomentar esse modelo de produção e distribuição de produtos agrícolas.

\section{Agradecimento}

Agradecemos o apoio financeiro da FAP-DF, à toda equipe de professores e bolsistas, à participação de voluntários e a disponibilidade da Rede CSA Brasília e a demais membros das CSAs-DF.

\section{Referências}

Alexander, C., et al. (2013). Uma Linguagem de Padrões. Porto Alegre: Bookman.

Bava, S. C. (2004). Tecnologia social e desenvolvimento local. Em Tecnologia Social: uma estratégia para o desenvolvimento. Rio de Janeiro: Fundação Banco do Brasil.

Callon, M. (1987). Society in the making: the study of technology as a tool for sociological analysis. In: BIJKER, Wiebe E. et al. Social Construction of technological systems. Cambridge: Cambridge University Press.

Dagnino, R., Brandão, F., \& Novaes, H. (2004). Sobre o marco analítico-conceitual da tecnologia social. Em Tecnologia Social: uma estratégia para o desenvolvimento. Rio de Janeiro: Fundação Banco do Brasil.

Lassance JR, A., \& Pedreira, J. (2004). Tecnologias sociais e políticas públicas. Em Tecnologia Social: uma estratégia para o desenvolvimento. Rio de Janeiro: Fundação Banco do Brasil.

Santos, M. (1992). Espaço e Método. São Paulo: Nobel. 3 ed.

Shedroff, N. (1999). Information Interaction Design: a unified field theory of design. Em Jacobson, R. E. (Ed.), Information design (pp: 267-292). Cambridge, MA: MIT Press.

Takeuchi, H., \& Nonaka, I. (2008). Gestão do Conhecimento. Editora: Bookman Companhia Ed.

Torres, C. L. (2017). Comunidade que Sustenta a Agricultura: a reaplicação da tecnologia social a partir dos casos pioneiros em Brasília. Dissertação (Mestrado). Brasília, Brasil: Universidade de Brasília 
Santos, S., Torres, C. L. \& Camara, R. | Concepção e desenvolvimento de Sistema Interativo para o aperfeiçoamento da gestão de Comunidades que Sustentam a Agricultura no Distrito Federal

\section{Sobre os autores}

Sofia Santos, Graduanda, UnB, Brasil <sofiaguerragcs@gmail.com>

Camila Lombardi Torres, Mestre, UnB, Brasil <camila.Iombardi@gmail.com>

Rogerio Camara, Doutor, UnB, Brasil <rogeriocamara@gmail.com> 